\title{
Melanoma subtypes demonstrate distinct PD-L1 expression profiles
}

\author{
Genevieve J Kaunitz', Tricia R Cottrell², Mohammed Lilo², Valliammai Muthappan³, Jessica Esandrio ', Sneha Berry ${ }^{1,4}$, \\ Haiying Xu', Aleksandra Ogurtsova ${ }^{1}$, Robert A Anders' ${ }^{2}$, Alexander H Fischer ${ }^{1}$, Stefan $\mathrm{Kraft}^{5}$, Meg R Gerstenblith ${ }^{6}$, \\ Cheryl L Thompson ${ }^{7}$, Kord Honda ${ }^{6}$, Jonathan D Cuda ${ }^{1,2}$, Charles G Eberhart ${ }^{2,3,4}$, James T Handa ${ }^{3}$, Evan J Lipson ${ }^{4}$ and \\ Janis M Taube $e^{1,2,4}$
}

PD-L1 expression in the tumor immune microenvironment is recognized as both a prognostic and predictive biomarker in patients with cutaneous melanoma, a finding closely related to its adaptive (IFN- $\gamma$-mediated) mechanism of expression. Approximately $35 \%$ of cutaneous melanomas express PD-L1, however, the expression patterns, levels, and prevalence in rarer melanoma subtypes are not well described. We performed immunohistochemistry for PD-L1 and CD8 on 200 formalin-fixed paraffin-embedded specimens from patients with acral $(n=16)$, mucosal $(n=36)$, uveal $(n=103)$, and chronic sun-damaged (CSD) ( $n=45)$ melanomas (24 lentigo maligna, 13 'mixed' desmoplastic, and 8 'pure' desmoplastic melanomas). CD8+ tumor-infiltrating lymphocyte (TIL) densities were characterized as mild, moderate, or severe, and their geographic association with PD-L1 expression was evaluated. Discrete lymphoid aggregates, the presence of a spindle cell morphology, and the relationship of these features with PD-L1 expression were assessed. PD-L1 expression was observed in $31 \%$ of acral melanomas, $44 \%$ of mucosal melanomas, $10 \%$ of uveal melanomas, and $62 \%$ of CSD melanomas $(P<0.0001)$. Compared to our previously characterized cohort of cutaneous melanomas, the proportion of PD-L1(+) tumors was lower in uveal $(P=0.0002)$ and higher in CSD $(P=0.0073)$ melanomas, while PD-L1 expression in the acral and mucosal subtypes was on par. PD-L1 expression in all subtypes correlated with a moderate-severe grade of CD8+ TIL (all, $P<0.003$ ), supporting an adaptive mechanism of expression induced during the host antitumor response. The tumor microenvironments observed in CSD melanomas segregated by whether they were the pure desmoplastic subtype, which showed lower levels of PD-L1 expression when compared to other CSD melanomas $(P=0.047)$. The presence of lymphoid aggregates was not associated with the level of PD-L1 expression, while PD-L1(+) cases with spindle cell morphology demonstrated higher levels of PD-L1 than those with a nested phenotype $(P<0.0001)$. Our findings may underpin the reported clinical response rates for anti-PD-1 monotherapy, which vary by subtype.

Laboratory Investigation (2017) 97, 1063-1071; doi:10.1038/labinvest.2017.64; published online 24 July 2017

Increased objective response rates (ORRs) to anti-PD-1/ PD-L1 monotherapy as well as improved progression-free survival and overall survival have been linked to PD-L1 expression in the tumor microenvironment (TME) in some studies. ${ }^{1-4}$ Studies from our group and others indicate that the major mechanism driving melanoma tumor cell PD-L1 expression is an association with tumor-infiltrating lymphocytes (TIL), consistent with an endogenous antitumor immune response that may be unleashed with anti-PD-1/ PD-L1 therapies. ${ }^{5}$ Such adaptive PD-L1 expression by melanoma is not dependent on BRAF, NRAS, or c-KIT mutational status. ${ }^{6,7}$

Most studies in melanoma patients that have examined the relationship between PD-L1 expression in the pretreatment TME and response to anti-PD-1/PD-L1 have focused on conventional cutaneous melanoma, ie, melanoma arising

\footnotetext{
${ }^{1}$ Department of Dermatology, Johns Hopkins University School of Medicine, Bloomberg Kimmel Institute for Cancer Immunotherapy, and Sidney Kimmel Comprehensive Cancer Center, Baltimore, MD, USA; ${ }^{2}$ Department of Pathology, Johns Hopkins University School of Medicine, Bloomberg Kimmel Institute for Cancer Immunotherapy, and Sidney Kimmel Comprehensive Cancer Center, Baltimore, MD, USA; ${ }^{3}$ Department of Ophthalmology, Johns Hopkins University School of Medicine, Bloomberg Kimmel Institute for Cancer Immunotherapy, and Sidney Kimmel Comprehensive Cancer Center, Baltimore, MD, USA; ${ }^{4}$ Department of Oncology, Johns Hopkins University School of Medicine, Bloomberg Kimmel Institute for Cancer Immunotherapy, and Sidney Kimmel Comprehensive Cancer Center, Baltimore, MD, USA; ${ }^{5}$ Department of Pathology, Massachusetts General Hospital, Boston, MA, USA; ${ }^{6}$ Department of Dermatology, University Hospitals Cleveland Medical Center/Case Western Reserve University School of Medicine, Case Comprehensive Cancer Center, Cleveland, OH, USA and ${ }^{7}$ Department of Nutrition, Case Western Reserve University School of Medicine, Cleveland, OH, USA Correspondence: Dr JM Taube, MD, Division of Dermatopathology, Johns Hopkins Hospital, Blalock 907, 600N. Wolfe Street, Baltimore, MD 21287, USA.

E-mail: jtaube1@jhmi.edu
}

Received 26 January 2017; revised 24 March 2017; accepted 28 April 2017 
on hair-bearing, non-chronically sun-damaged skin. Approximately two-thirds of these melanomas harbor BRAF mutations, and are most often classified histologically as superficial spreading and nodular melanomas. ${ }^{8}$ Chronic sun damage (CSD) melanomas tend to occur in an older demographic on the head and neck, and include the histologic categories of lentigo maligna melanoma and desmoplastic melanoma. Desmoplastic melanoma may be further subdivided into the pure and mixed (ie, a 'mix' of both desmoplastic and conventional-appearing melanoma) categories, with pure desmoplastic melanomas being defined morphologically as those comprised of $>90 \%$ sparse, spindled melanocytes set in a dense, fibrocollagenous stroma resembling a scar. ${ }^{9}$ This subtype is also often associated with intratumoral lymphoid aggregates and is more likely to be associated with mutations in tumor-suppressor genes when compared to the mixed desmoplastic type, which is more likely to be associated with BRAF and NRAS activating oncogenic driver mutations. ${ }^{10}$ Acral melanomas arise on the non-hair-bearing skin of the palms and soles; mucosal melanomas arise from melanocytes of the mucosal epithelium of the paranasal sinuses/oropharynx or anogenital region; and uveal melanomas arise from nonepithelial melanocytes in the dermis or uvea. UV radiation is not a dominant mutagen in these latter three types (for a thorough review of melanoma subtype classification see Whiteman, et $a l^{8}$ ).

A few recent studies have reported objective response rates to anti-PD-1/PD-L1 in patients with mucosal, acral, uveal, and desmoplastic melanomas (summarized in Table 1). ORRs for patients with uveal melanoma were notably lower than for patients with cutaneous melanoma, while patients with desmoplastic melanoma demonstrated higher response rates. Most of these studies did not include immunohistochemistry (IHC) assessment of PD-L1 expression in pretreatment surgical pathology specimens, thus it is unclear how PD-L1 expression patterns in these tumor types may relate to the observed response rates. The purpose of our study was to characterize PD-L1 expression prevalence, patterns, and levels among these melanoma variants.

\section{MATERIALS AND METHODS Case Selection}

Following IRB approval, 200 archival surgical pathology specimens from 195 distinct patients were collected from three different academic medical centers (Johns Hopkins University, Baltimore, MD; Massachusetts General Hospital, Boston, MA; and University Hospitals Cleveland Medical Center, Cleveland, $\mathrm{OH}$ ). The cohort included 16 acral melanomas, 36 mucosal melanomas, 103 uveal melanomas, and 45 chronic sun damage (CSD) melanomas, including 24 lentigo maligna and 21 desmoplastic melanomas. ${ }^{11}$ Eighty of the uveal melanomas were studied in a tissue microarray (TMA) format. A summary of specimen origin (ie, primary lesion vs metastasis) and histologic subtype is provided in Table 2. The histologic diagnosis for each specimen was confirmed on H\&E slides by a dermatopathologist (JMT) or an ophthalmic pathologist (CE), and a single representative tissue block was chosen for additional study. Morphologic features were tabulated, including the presence of a spindled or nested morphology, lymphoid aggregates (clusters of $>25$ lymphocytes with or without macrophages or dendritic cells), and when relevant, the pure $v s$ mixed desmoplastic features. ${ }^{9}$

Table 1 Response to anti-PD-1/PD-L1 therapy in melanoma subtypes

\begin{tabular}{|c|c|c|c|c|c|}
\hline Melanoma subtype & Study & ORR (\%) & Total no. patients & Oncologic agent & Reference \\
\hline \multirow[t]{3}{*}{ Acral } & 1 & 32 & 25 & Anti-PD-1 & Shoushtari et al ${ }^{52}$ \\
\hline & 2 & 16.7 & 6 & Anti-PD-1 & Cho et $a p^{53}$ \\
\hline & Overall & 29.0 & 31 & & \\
\hline \multirow[t]{4}{*}{ Mucosal } & 1 & 23 & $35^{a}$ & Anti-PD-1 & Shoushtari et al ${ }^{52}$ \\
\hline & 2 & 20 & $5^{b}$ & Anti-PD-L1 & Herbst et $\left.a\right|^{54}$ \\
\hline & 3 & 23.3 & $86^{b}$ & Anti-PD-1 & $D^{\prime}$ Angelo et $\left.a\right|^{51}$ \\
\hline & Overall & 23.0 & 126 & & \\
\hline \multirow[t]{4}{*}{ Uveal } & 1 & 37.5 & 8 & Anti-PD-1 & Kottschade et $a^{55}$ \\
\hline & 2 & 8 & 25 & Anti-PD-1 & Karydis et al ${ }^{18}$ \\
\hline & 3 & 3.6 & 56 & Anti-PD-1/PD-L1 & Algazi et al ${ }^{56}$ \\
\hline & Overall & 7.9 & 89 & & \\
\hline $\mathrm{CSD}^{\mathrm{C}}$ & 1 & 70 & 23 & Anti-PD-1/PD-L1 & Eroglu et al ${ }^{49}$ \\
\hline
\end{tabular}

\footnotetext{
${ }^{a} 12(35 \%)$ patients had anorectal disease, 14 (40\%) had vulvovaginal, and 9 (26\%) had head/neck. ORR was not reported for specific anatomic sites. ${ }^{b}$ Specific anatomic sites for the primary tumor were not delineated.

${ }^{c}$ CSD, chronic sun damage. ${ }^{11}$ Term includes desmoplastic (pure and mixed) and lentigo maligna melanoma, and subtype not-specified on sun-damaged skin. The associated study reported ORR for pure and mixed desmoplastic types, and subtype not-specified.
} 
Table 2 Characteristics of archival surgical pathology specimens studied from patients with various melanoma subtypes

No. specimens, $n(\%) \quad$ Primary ${ }^{\mathrm{a}}, n$ Metastatic, $n$

\begin{tabular}{lccc} 
Total & $\mathbf{2 0 0 ( 1 0 0 )}$ & $\mathbf{1 9 4}$ & $\mathbf{6}$ \\
Acral & $\mathbf{1 6 ( 8 )}$ & $\mathbf{1 6}$ & $\mathbf{0}$ \\
Mucosal & $\mathbf{3 6 ( 1 8 )}$ & $\mathbf{3 3}$ & $\mathbf{3}$ \\
$\quad$ Anorectal & $3(1.5)$ & 2 & 1 \\
$\quad$ Vulvovaginal & $7(3.5)$ & 7 & 0 \\
$\quad$ Sinonasal & $26(13)$ & 24 & 2 \\
Uveal & $103(51.5)$ & 101 & $\mathbf{2}$ \\
CSD & $\mathbf{4 5 ( 2 2 . 5 )}$ & $\mathbf{4 4}$ & $\mathbf{1}$ \\
$\quad$ Pure desmoplastic & $8(4)$ & 8 & 0 \\
$\quad$ Mixed desmoplastic & $13(6.5)$ & 12 & 1 \\
Lentigo maligna & $24(12)$ & 24 & 0 \\
\hline
\end{tabular}

${ }^{\mathrm{a}}$ Fourteen primary tumor specimens ( 3 mucosal, 1 uveal, 8 CSD, and 2 acral) were excisions containing recurrent/residual disease, as opposed to a primary diagnostic biopsy.

Bold values indicate the overall ORR and total \# patients in each category.

For the purpose of this study, the terms 'spindled' or 'spindle cell' are used as morphologic descriptors, and not as a distinct melanoma subtype.

\section{IHC Studies and Characterization of TIL Patterns}

IHC for PD-L1 expression was performed using the 5H1 antibody as previously described. ${ }^{5} \mathrm{~A}$ modified counterstain was used for the uveal and other heavily pigmented melanomas. ${ }^{12}$ Specifically, Giemsa was substituted for hematoxylin to enhance differentiation between the brown chromogen signal used in the IHC method from the native melanin pigment. IHC for CD8 was also performed on each case using standard automated methods.

PD-L1 expression was observed on both tumor cells and immune cells, and was differentiated based on morphologic features and geographic distribution. PD-L1 expression on tumor cells was scored as $0 \%, 5 \%, 10 \%$, and at increasing $10 \%$ intervals. ${ }^{5,13}$ Tumors were considered to be PD-L1 $(+)$ when $\geq 5 \%$ of tumor cells demonstrated membranous staining. ${ }^{5,13}$ The presence of CD8+ TIL was scored as 0 (none), mild (1, positive cells focally at advancing edge of tumor or perivascularly, $<5 \%$ of tumor area), moderate ( 2 , infiltrating tumor by extending away from intratumoral vessels or extending into tumor from advancing edge, $5-50 \%$ of tumor area), or severe (3, marked, broad infiltration by TIL or a strong immune front, $>50 \%$ of tumor area). The TIL intensity grading system has been shown to correlate with TIL counts by digital image analysis in previous studies. ${ }^{14,15}$ For the fourteen re-excision specimens that included a primary biopsy site, PD-L1 expression and TIL densities were assessed away from the primary biopsy location. The correlations between PD-L1 expression with CD8+ TIL infiltrates, discrete lymphoid aggregates, and spindle (fusiform) cell morphology were assessed.

\section{Statistical Analysis}

The chi-square test was used to assess differences in the proportion of $\mathrm{PD}-\mathrm{L} 1(+)$ tumors among all melanoma subtypes. The Kruskal-Wallis test was used to compare the levels of PD-L1 expression across all subtypes. The Fisher's exact test was used to compare the proportion of samples with CD8+ T-cell infiltration in PD-L1(+) and PD-L1(- ) samples. Comparisons between PD-L1 expression levels and morphologic characteristics (pure desmoplastic melanoma $v s$ other CSD melanomas, presence or absence of lymphoid aggregates, and spindled $v s$ nested morphology) were calculated using the Mann-Whitney $U$-test. Statistical significance was determined using two-tailed tests with an alpha level of 0.05 unless otherwise stated. Statistical analyses were performed with GraphPad Prism 7 (GraphPad Software) and Stata 14.1 (StataCorp, College Station, TX, USA).

\section{RESULTS}

\section{PD-L1 Expression Patterns, Prevalence, and Expression Levels}

PD-L1 tumor cell expression was observed in 30\% (60/200) of specimens studied. When present, PD-L1 expression on tumor cells was predominantly observed in geographic association with CD8+ T-lymphocytes, consistent with an adaptive mechanism of expression, Figure 1a. This pattern was most apparent when moderate to severe intensities of CD8+ lymphocytes were present as compared to mild or none, and was observed in all histologic subtypes, Figure $1 \mathrm{~b}$. Constitutive PD-L1 expression (tumor cell PD-L1 expression with no TIL) was not observed, although PD-L1 was expressed on immune cells, including lymphocytes and macrophages, without adjacent tumor cell expression.

We previously reported PD-L1 expression in 35\% of cutaneous melanoma samples from 54 patients, when a threshold of $\geq 5 \%$ tumor cell expression was used. ${ }^{5}$ Among the more rare melanoma subtypes studied here using the same method, the proportion of $\mathrm{PD}-\mathrm{L} 1(+)$ tumors varied significantly $(P<0.0001)$. When compared to cutaneous melanoma, uveal melanomas demonstrated the lowest proportion of PD-L1 expression, at 10\% $(P=0.0002)$, while the highest proportion of PD-L1(+) specimens was seen in the CSD melanomas, at $62 \%(P=0.0073)$. The proportion of acral and mucosal melanomas expressing PD-L1 was comparable to cutaneous disease, at 31 and $44 \%(P=0.77$ and 0.38 , respectively), Figure $2 \mathrm{a}$. We next examined whether there were differences in the median or range of PD-L1 expression levels by melanoma subtype among the PD-L1(+) specimens. We found that the median levels were similar across subtypes, although acral melanomas seemed to show a lower range of expression, Figure 2b. 


\section{Association of PD-L1 Expression with Morphologic Features}

Discrete, well-formed lymphoid aggregates were observed in $75 \%(6 / 8)$ of pure desmoplastic melanomas, as well as in $22 \%$ $(8 / 37)$ of other CSD melanoma specimens. Lymphoid aggregates were not a prominent feature in the other melanoma subtypes studied. PD-L1 was expressed by macrophages and dendritic cells as well as by lymphocytes in these aggregates, Figure 3a. In the $62 \%(5 / 8)$ of PD-L1(+) pure desmoplastic melanomas, PD-L1 expression was observed immediately adjacent to the lymphoid aggregates, and did not exceed a 5\% tumor expression level. PD-L1(+) pure desmoplastic melanomas displayed lower median levels of PD-L1 expression as compared to other CSD melanomas $(P=0.047)$, Figure 3b, Figure 4a. This is in keeping with recent results showing that $\mathrm{PD}-\mathrm{L} 1$ expression is lower in pure vs mixed desmoplastic melanomas. ${ }^{16} \mathrm{PD}-\mathrm{L} 1$ expression levels among CSD melanomas were not influenced by the presence of lymphoid aggregates, Figure $4 \mathrm{~b}$.

Some of the highest PD-L1 expression levels were seen in CSD melanomas. When we examined those specimens demonstrating higher levels of expression, they were often the mixed desmoplastic subtype with a prominent spindle cell morphology, rather than the pure desmoplastic subtype.
Across all the subtypes, the only other specimen with $>50 \%$ expression was a mucosal melanoma specimen, which also had a spindled configuration, Figure $2 \mathrm{~b}$. When the relationship among melanomas expressing PD-L1 and a spindled $v s$ nested morphology was assessed, there was a statistically significant correlation between higher levels of PD-L1 expression and a spindled configuration (median PD-L1 expression was $50 \%$ for cases with a spindled morphology $v s$ $5 \%$ for nested; $P<0.0001$ ), Figure $4 \mathrm{c}$.

\section{DISCUSSION}

Cancers may express PD-L1 to form a so-called 'molecular shield' against antitumor immune effector cells, facilitating immune evasion. ${ }^{17}$ When TILs recognize tumors, they secrete IFN- $\gamma$ and other pro-inflammatory cytokines, which can upregulate PD-L1 expression in the TME. PD-L1, in turn, may ligate PD-1 on TIL triggering their downregulation. ${ }^{18}$ Adaptive PD-L1 expression in the pretreatment melanoma microenvironment has been associated with an improved prognosis, ${ }^{5}$ and is also predictive of response to anti-PD-1/ PD-L1 therapies in melanoma and other tumor types. ${ }^{13,19-21}$ Most of the early clinical trials of anti-PD-1/PD-L1 immunotherapies that evaluated PD-L1 as a biomarker of response excluded patients with non-cutaneous melanomas,
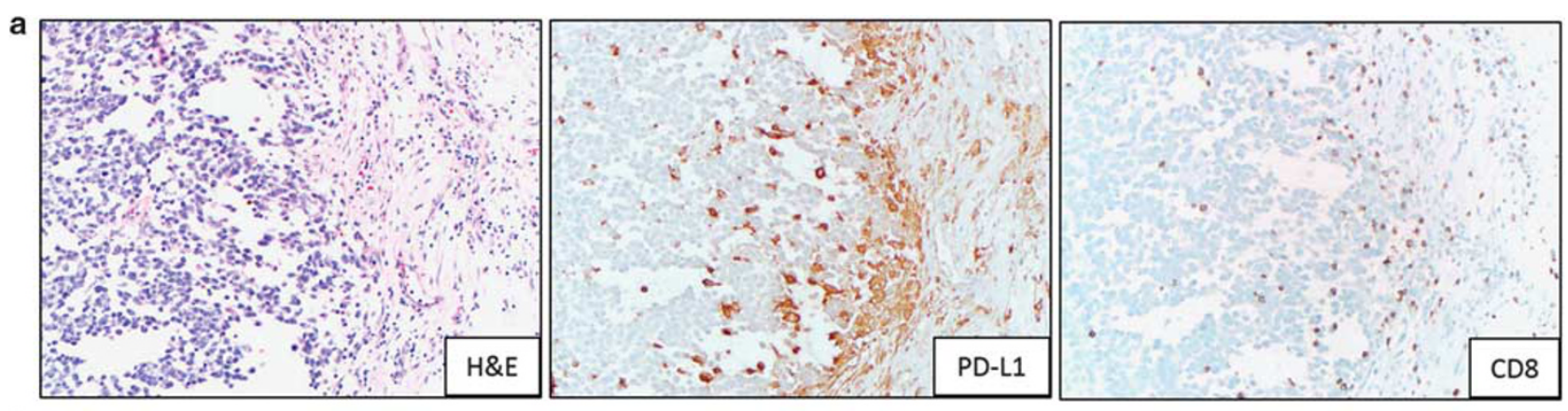

\begin{tabular}{|c|c|c|c|c|c|}
\hline \multirow{2}{*}{ Melanoma subtype (n) } & \multicolumn{2}{|c|}{ PD-L1(+) } & \multicolumn{2}{|c|}{ PD-L1(-) } & \multirow{2}{*}{ p-value ${ }^{c}$} \\
\hline & CD8 TIL ${ }^{\text {high b }}$ & CD8 TIL low & CD8 TIL ${ }^{\text {high }}$ & CD8 TIL low & \\
\hline acral (16) & 4 & 1 & 0 & 11 & 0.0027 \\
\hline mucosal (36) & 15 & 1 & 1 & 19 & $<0.0001$ \\
\hline uveal (103) & 10 & 1 & 1 & 91 & $<0.0001$ \\
\hline $\operatorname{CSD}(45)$ & 21 & 7 & 0 & 17 & $<0.0001$ \\
\hline
\end{tabular}

Figure 1 All melanoma subtypes demonstrate PD-L1 expression in geographic association with CD8+ tumor-infiltrating lymphocytes (TIL), consistent with an adaptive (IFN- $\gamma$-mediated) mechanism of expression. (a) PD-L1 expression on both tumor cells and immune cells is observed at the tumor-host interface in a vulvar mucosal melanoma. 200x original magnification, all panels. (b) The adaptive pattern of expression was consistently observed across all melanoma subtypes studied. There were only two melanomas studied with moderate-severe grade of TIL present that lacked PD-L1 expression (2/54, 4\%). 

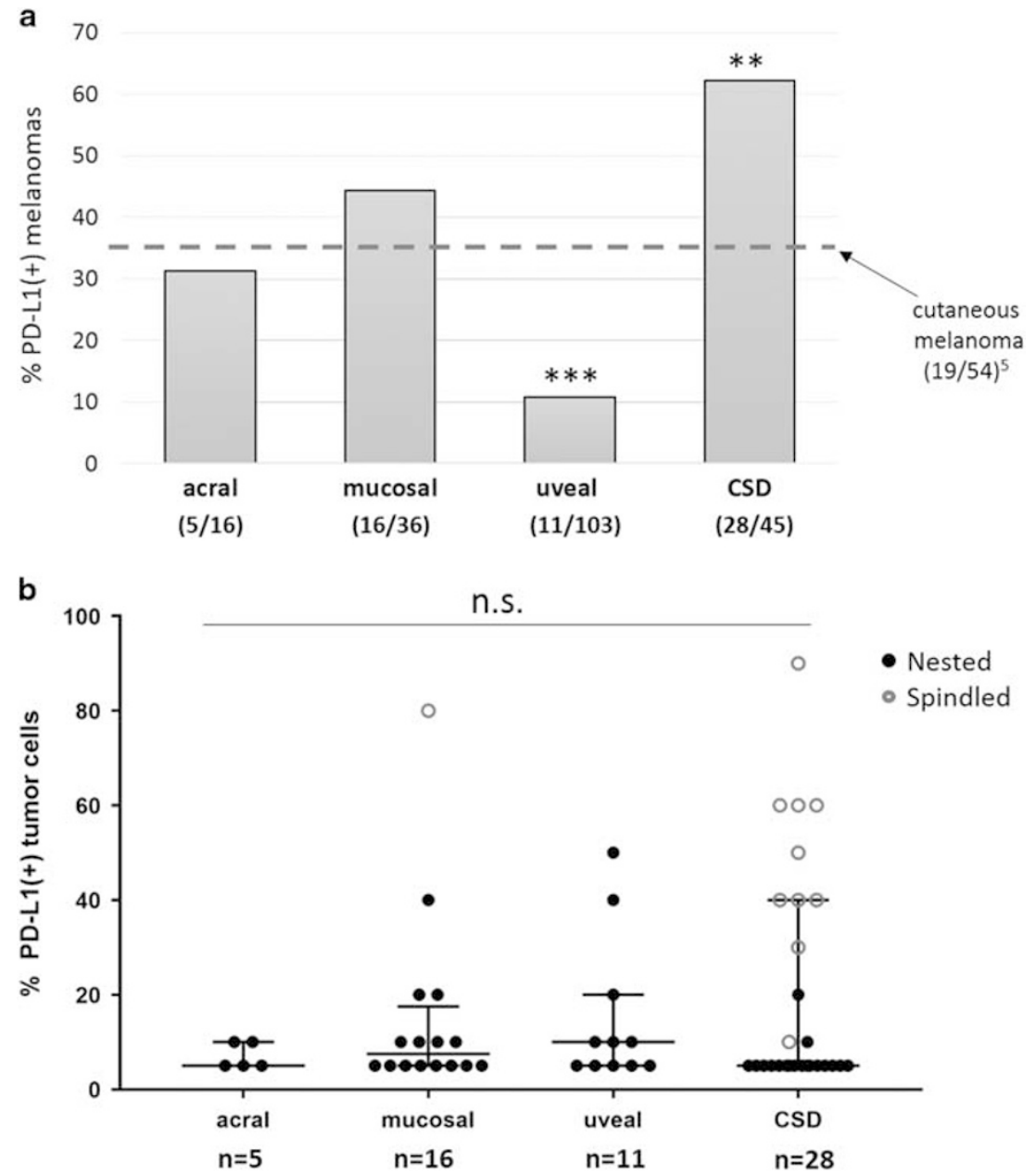

Figure 2 Prevalence and levels of PD-L1 expression vary by histologic subtype of melanoma. (a) Uveal melanomas showed the lowest proportion of cases with PD-L1 expression, while CSD melanomas showed the highest proportion. The proportion of PD-L1(+) tumors in both of these subtypes was significantly different from conventional cutaneous melanoma $\left({ }^{* *} P=0.0002\right.$ and ${ }^{*} P=0.0073$, chi-square test), the latter reported previously using the same staining and scoring methods. ${ }^{5}$ The proportion of PD-L1(+) cases among acral and mucosal melanomas was not significantly different from that observed in cutaneous disease. (b) PD-L1 expression levels were compared by melanoma subtype in cases that were PD-L1(+). A narrower range of expression levels was observed in acral melanomas when compared to the other subtypes, but significant differences in the median levels of PD-L1 expression were not observed $(P=0.91)$. n.s., not significant.

or did not distinguish between distinct histologic subtypes. Many of the more recent studies reporting response rates to anti-PD-1/PD-L1 for patients with melanoma variants did not study PD-L1 expression. We present here a detailed characterization of patterns, prevalence, and levels of PD-L1 expression in four distinct subtypes of melanoma.

We found that the proportion of $\mathrm{PD}-\mathrm{L} 1(+)$ cases by melanoma subtype generally tracked the reported ORRs to anti-PD-1/PD-L1 (Table 1). In our study, $62 \%$ of CSD melanomas were PD-L1(+) compared to $31 \%$ of acral melanomas, $44 \%$ of mucosal melanomas, and $10 \%$ of uveal melanomas. In a previous study, 35\% of cutaneous melanomas were PD-L1(+) using a similar IHC method and scoring system. Reported overall ORRs for these subtypes range from $71 \%$ for desmoplastic (pure or mixed) melanoma to $8 \%$ for uveal melanoma, while ORRs in acral and mucosal melanomas are intermediate, at 29 and 23\%, respectively. Patients with advanced conventional cutaneous melanoma show ORR on the order of $30-40 \%,{ }^{19,22-29}$ which varies depending on whether the agents are given in the first line or as later-line therapy. Importantly, while the proportion of PD-L1(+) tumors in these subtypes appears to generally parallel the reported ORR on a population level, PD-L1 as a biomarker has limitations on a per-patient basis. Indeed, patients whose tumors are PD-L1(+) may not respond, while $\sim 15 \%$ of patients with PD-L1(-) tumors demonstrate an antitumor response to therapy. ${ }^{1}$

While tumor cell PD-L1 expression varied among melanoma subtypes, we found that when present, it was geographically associated with CD8+ T-cells, similar to most conventional cutaneous melanomas. Previous studies have supported the presence of a possible IFN- $\gamma$-mediated adaptive 


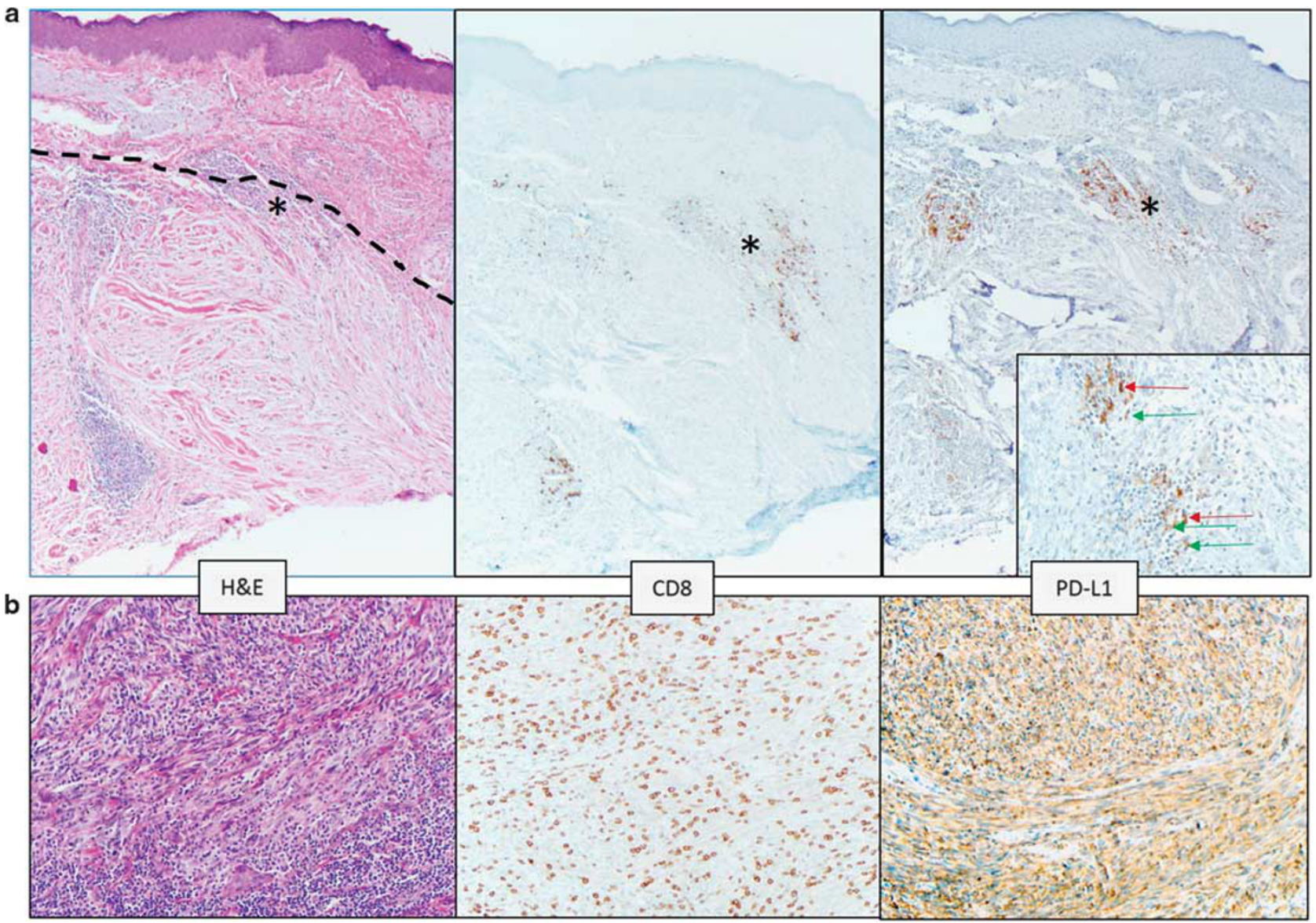

Figure 3 Pure desmoplastic melanomas demonstrate a different PD-L1 expression pattern than other CSD melanomas. (a) The dotted line in the left panel delineates the upper boundary of a desmoplastic melanoma centered in the dermis. Solar elastosis is a prominent feature in the superficial dermis. Lymphoid aggregates are present, and one at the boundary of the melanoma and normal dermis is marked with an asterisk (*) and is shown on the inset of the right panel. In the pure desmoplastic melanomas, PD-L1 expression was observed on lymphocytes (green arrow on inset) and macrophages (red arrows on inset) in lymphoid aggregates. This case did not show PD-L1 expression on tumor cells. Original magnification, $\times 100$, inset $\times 400$. (b) CSD melanoma with spindled morphology showing $60 \%$ tumor cell PD-L1 expression associated with a 'severe' grade CD8+ TIL infiltrate. 'Severe' grade is defined as a diffuse infiltrate of TIL throughout the melanoma. $\times 200$ original magnification.

a

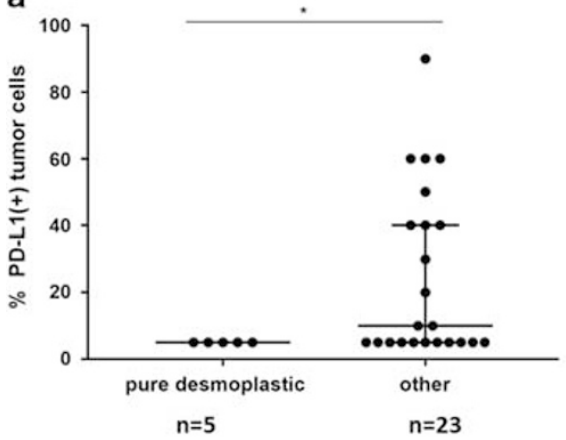

b

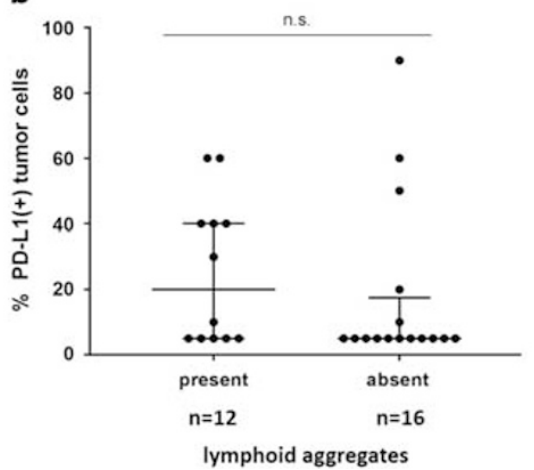

C

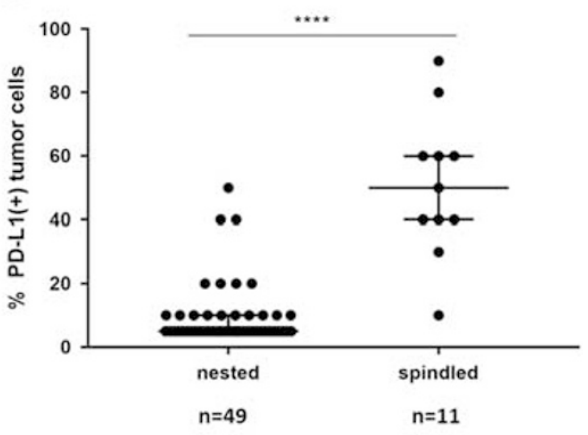

Figure 4 Association of morphologic features with levels of PD-L1 expression. (a) Among PD-L1(+) CSD melanomas, there were significantly higher median PD-L1 expression levels in cases lacking a pure desmoplastic phenotype ${ }^{*} P=0.047$, Mann-Whitney $U$-test). (b) The presence or absence of lymphoid aggregates did not correlate with the levels of PD-L1 expression observed in PD-L1(+) CSD melanomas $(P=0.22)$. (c) Among all melanoma subtypes, PD-L1(+) tumors with a spindled morphology demonstrated a higher percentage of tumor cells with PD-L1 display compared to those with a nested morphology $(* * * *<<0.0001)$. n.s., not significant. 
mechanism of PD-L1 display in uveal melanoma cell lines. ${ }^{30}$ The association between PD-L1 expression and TILs is also in keeping with a prior observation that the presence of TILs is associated with improved outcomes in acral melanoma ${ }^{31}$ and a lower risk of metastasis in mucosal melanoma, ${ }^{32}$ and that patients with $\mathrm{PD}-\mathrm{L} 1(+)$ mucosal melanoma experience a significantly longer recurrence-free survival. ${ }^{33}$

CSD melanomas showed two distinct patterns of PD-L1 display. In cases of pure desmoplastic melanoma, PD-L1 expression by tumor cells was low, and in fact was only observed on tumor cells immediately surrounding the characteristic lymphoid aggregates. In contrast, those that were the mixed desmoplastic type or lentigo maligna type showed some of the highest levels of PD-L1 expression. In each of these latter cases, PD-L1 expression was associated with a diffuse $\mathrm{CD} 8+\mathrm{T}$-cell infiltrate, extending throughout the tumor. Notably, the only non-CSD melanoma case with $>50 \%$ PD-L1 expression also had a spindled morphology and a moderate-severe TIL infiltrate. Spindle cell morphology in melanomas is thought to represent a more immature, dedifferentiated, mesenchymal phenotype. ${ }^{34,35} \mathrm{PD}-\mathrm{L} 1$ expression has previously been associated with an epithelial to mesenchymal transition (EMT) in other tumor types, ${ }^{36,37}$ though the relationship between PD-L1, EMT, and the presence of a cytotoxic T-cell infiltrate was not assessed. Our findings comparing the pure desmoplastic type $v s$ other CSD melanomas raise the possibility that it could be a combination of EMT (as exemplified here by a spindle cell morphology) and a diffusely-arrayed antitumor immune response that may contribute to increased levels of PD-L1 expression.

Features beyond PD-L1 expression such as mutational burden have also been proposed as biomarkers of response to anti-PD-1. ${ }^{38,39}$ Among all melanomas, CSD melanomas have the highest mutational burden (median 62 mutations/ megabase $(\mathrm{Mb})$ ), with $\mathrm{UV}$ radiation serving as the dominant mutagen. ${ }^{40}$ Cutaneous melanoma has a mutation rate of $\sim 15$ mutations/Mb, ${ }^{41,42}$ while the somatic mutation rate in acral and mucosal melanomas is 5-10-fold lower. ${ }^{43,44}$ Uveal melanomas have the lowest mutational burden of any melanoma subtype..$^{45}$ Thus, mutational burden also generally parallels the reported ORRs for melanoma subtypes, though perhaps not as closely as PD-L1 expression. It would be anticipated that if mutational burden were the dominant factor predicting response to therapy, mucosal and acral melanomas would have a proportionately lower response rate than cutaneous melanomas, which is not the case. A recent study showed that mutational burden was a contributing factor to general prognosis for patients with melanoma, but its relative contribution was less than PD-1/PD-L1 axis molecule expression. ${ }^{7}$ It is possible that a similar relationship between these factors also exists when predicting therapeutic response to anti-PD-1, ie, mutational burden is a contributing factor to response, but is subdominant to PD-L1 expression. More recent studies have identified chromosomal alterations as contributing genetic factors of resistance to immunotherapy. ${ }^{46,47}$ The relationship of these alterations to PD-L1 expression or specific melanoma subtypes has yet to be defined.

Pure desmoplastic melanomas tend to be characterized by the presence of lymphoid aggregates. Such intratumoral lymphoid aggregates have both prognostic and therapeutic value, as they facilitate lymphocyte recruitment and help maintain both T-cell and B-cell antitumor responses. ${ }^{48} \mathrm{We}$ found similar proportions of PD-L1(+) tumors in both pure desmoplastic melanoma and other CSD melanomas, though the latter showed significantly higher levels of expression. An early report studying patients with desmoplastic melanomas receiving anti-PD-1 showed similar response rates for the pure and mixed variants. ${ }^{49}$ It is possible that responses in the pure variant may be facilitated by the lymphoid aggregates, as well as by the presence of a high mutational load. This highlights one of the limitations of this study, which is that the patients included in the analysis were not treated with anti-PD-1; the relationships proposed here between lymphoid aggregates and the levels, prevalence, and patterns of PD-L1 expression with the anti-PD-1 response are based on historical response rates.

Other potential limitations of this study include sampling and the stage of lesions studied, as well as the use of a threshold to dichotomize PD-L1 expression. PD-L1 expression may be focal and geographic. We have previously validated the use of TMAs for studying PD-L1 expression in squamous cell carcinoma and showed 85\% concordance in tumor cell PDL1 expression between tumor cores and whole-mount sections, ${ }^{15}$ but it is possible that requisite sampling ratios may vary by tumor type. Thus the reported rates of PD-L1 expression may be underestimated here in uveal melanomas, where we used TMAs to study a significant proportion of cases. We did not characterize a broad immune cell repertoire here, which represents another possible limitation. Cell types such as regulatory T-cells and myeloid-derived suppressor cells may have a suppressive effect on the host immune response. In addition, the tumors studied here were predominantly primary lesions, while most patients treated with anti-PD-1 have metastatic disease. Discordance exists in PD-L1 expression between primary and metastatic lesions from the same patient as a result of the focal and dynamic nature of this biomarker. ${ }^{3,13,19}$ Many of the early clinical trials that studied the relationship between PD-L1 expression in the pretreatment TME and response to anti-PD-1 did not specifically require an immediate pretreatment sample from a metastasis for the assessment of PD-L1 status. In a number of cases, primary melanomas or archival samples from metastases taken years prior to treatment were assayed. ${ }^{13,19,23,25}$ It remains to be determined whether a specimen from a metastasis obtained immediately prior to treatment may have improved predictive value over a primary tumor sample when determining the response to therapy in the metastatic setting. The predictive value of $\mathrm{PD}-\mathrm{L} 1$ expression for the treatment of non-resectable, aggressive primary melanomas, eg, some CSD melanomas, will 
also require separate, focused analysis. Lastly, PD-L1 may be considered to demonstrate a continuous range of expression, yet patients are often dichotomized into classes of PD-L1(+) vs PD-L1(-). It is likely that our reported PD-L1 expression prevalences across melanoma subtypes would change if a different threshold for PD-L1 positivity were used (ie, 1 vs $5 \%$ ).

In summary, our findings highlight that PD-L1 expression varies by melanoma subtype. Patients with CSD melanomas had the highest proportion of PD-L1(+) tumors and PD-L1 expression levels. These features, when combined with other characteristics of the tumor microenvironment, such as high mutational load and the presence of lymphoid aggregates, provide a possible explanation for the observed high response rates to anti-PD-1 monotherapy in these patients. By contrast, uveal melanomas demonstrate the lowest TIL densities and PD-L1 positivity, which may be due to the fact they have the least genomic instability and that the eye is an immunologically privileged site. ${ }^{50}$ As such, our findings also provide an explanation for low observed response rates to anti-PD-1 monotherapy in this melanoma subtype and support ongoing clinical trials exploring dual anti-CTLA-4/PD-1 blockade (NCT02626962) potentially combined with radiation (NCT02913417). Patients with acral and mucosal melanomas lacking PD-L1 expression may also benefit from dual antiCTLA-4/PD-1 blockade or other combinatorial immunotherapeutic regimens, ${ }^{51}$ similar to patients with cutaneous melanoma.

\section{ACKNOWLEDGMENTS}

We thank Dr Suzanne L. Topalian (Johns Hopkins University School of Medicine) for critical review of the manuscript. This work was supported by the Dermatology Foundation (JMT, MRG); the Melanoma Research Alliance (JMT); Bristol-Myers Squibb (JMT, RAA); Sidney Kimmel Cancer Center Core Grant P30 CA006973 (JMT, EJL); the National Cancer Institute NIH Grant R01 CA142779 (JMT); NIH Grant T32 CA193145 (TRC); unrestricted grant from Research to Prevent Blindness (Wilmer Eye Institute) (JTH); Moving for Melanoma of Delaware (JMT, EJL); and the Char and Chuck Fowler Family Foundation (MRG, CLT). We were also supported by the Bloomberg Kimmel Institute for Cancer Immunotherapy and a Stand Up To Cancer-Cancer Research Institute Cancer Immunology Translational Cancer Research Grant (SU2C-AACR-DT1012). Stand Up To Cancer is a program of the Entertainment Industry Foundation administered by the American Association for Cancer Research.

\section{DISCLOSURE/CONFLICT OF INTEREST}

RAA receives research funding from Five Prime Therapeutics and Bristol-Myers Squibb. JTH received research funding from Bayer Pharmaceuticals, Inc. EJL is an advisory board member/consultant for Bristol-Myers Squibb, EMD Serono, Merck and Novartis, and receives research funding from Merck. JMT is an advisory board member/consultant for Bristol-Myers Squibb, Astra-Zeneca and Merck, and receives research funding from Bristol-Myers Squibb. The remaining authors declare no conflict of interest.

1. Sunshine J, Taube JM. PD-1/PD-L1 inhibitors. Curr Opin Pharmacol 2015;23:32-38.

2. Daud Al, Wolchok JD, Robert C, et al. Programmed death-ligand 1 expression and response to the anti-programmed death 1 antibody pembrolizumab in melanoma. J Clin Oncol 2016;34:4102-4109.
3. Madore J, Vilain RE, Menzies AM, et al. PD-L1 expression in melanoma shows marked heterogeneity within and between patients: implications for anti-PD-1/PD-L1 clinical trials. Pigment Cell Melanoma Res 2015;28:245-253.

4. Obeid JM, Erdag G, Smolkin ME, et al. PD-L1, PD-L2 and PD-1 expression in metastatic melanoma: Correlation with tumor-infiltrating immune cells and clinical outcome. Oncoimmunology 2016;5:e1235107.

5. Taube JM, Anders RA, Young GD, et al. Colocalization of inflammatory response with B7-h1 expression in human melanocytic lesions supports an adaptive resistance mechanism of immune escape. Sci Transl Med 2012;4:127ra137.

6. Rodic N, Anders RA, Eshleman JR, et al. PD-L1 expression in melanocytic lesions does not correlate with the BRAF V600E mutation. Cancer Immunol Res 2015;3:110-115.

7. Danilova L, Wang H, Sunshine J, et al. Association of PD-1/PD-L axis expression with cytolytic activity, mutational load, and prognosis in melanoma and other solid tumors. Proc Natl Acad Sci USA 2016;113: E7769-E7777.

8. Whiteman DC, Pavan WJ, Bastian BC. The melanomas: a synthesis of epidemiological, clinical, histopathological, genetic, and biological aspects, supporting distinct subtypes, causal pathways, and cells of origin. Pigment Cell Melanoma Res 2011;24:879-897.

9. Busam KJ, Mujumdar U, Hummer AJ, et al. Cutaneous desmoplastic melanoma: reappraisal of morphologic heterogeneity and prognostic factors. Am J Surg Pathol 2004;28:1518-1525.

10. Jahn SW, Kashofer K, Halbwedl I, et al. Mutational dichotomy in desmoplastic malignant melanoma corroborated by multigene panel analysis. Mod Pathol 2015;28:895-903.

11. Curtin JA, Fridlyand J, Kageshita $T$, et al. Distinct sets of genetic alterations in melanoma. N Engl J Med 2005;353:2135-2147.

12. Kamino H, Tam ST. Immunoperoxidase technique modified by counterstain with azure B as a diagnostic aid in evaluating heavily pigmented melanocytic neoplasms. J Cutan Pathol 1991;18:436-439.

13. Taube JM, Klein A, Brahmer JR, et al. Association of PD-1, PD-1 ligands, and other features of the tumor immune microenvironment with response to anti-PD-1 therapy. Clin Cancer Res 2014;20:5064-5074.

14. Cimino-Mathews A, Thompson E, Taube JM, et al. PD-L1 (B7-H1) expression and the immune tumor microenvironment in primary and metastatic breast carcinomas. Hum Pathol 2016;1:52-63.

15. Yanik EL, Kaunitz GJ, Cottrell TR, et al. Association of HIV status with local immune response to anal squamous cell carcinoma: implications for immunotherapy. JAMA Oncol 2017; doi: 10.1001/ jamaoncol.2017.0115 (e-pub ahead of print).

16. Frydenlund N, Leone D, Yang S, et al. Tumoral PD-L1 expression in desmoplastic melanoma is associated with depth of invasion, tumorinfiltrating CD8 cytotoxic lymphocytes and the mixed cytomorphological variant. Mod Pathol 2017;30:357-369.

17. Dong H, Strome SE, Salomao DR, et al. Tumor-associated B7-H1 promotes T-cell apoptosis: a potential mechanism of immune evasion. Nat Med 2002;8:793-800.

18. Karydis I, Chan PY, Wheater M, et al. Clinical activity and safety of Pembrolizumab in Ipilimumab pre-treated patients with uveal melanoma. Oncoimmunology 2016;5:e1143997.

19. Topalian SL, Hodi FS, Brahmer JR, et al. Safety, activity, and immune correlates of anti-PD-1 antibody in cancer. N Engl J Med 2012;366: 2443-2454.

20. Tumeh PC, Harview CL, Yearley JH, et al. PD-1 blockade induces responses by inhibiting adaptive immune resistance. Nature 2014;515: 568-571.

21. Topalian SL, Taube JM, Anders RA, et al. Mechanism-driven biomarkers to guide immune checkpoint blockade in cancer therapy. Nat Rev Cancer 2016;16:275-287.

22. Ribas A, Puzanov I, Dummer $\mathrm{R}$, et al. Pembrolizumab versus investigator-choice chemotherapy for ipilimumab-refractory melanoma (KEYNOTE-002): a randomised, controlled, phase 2 trial. Lancet Oncol 2015;16:908-918.

23. Robert C, Ribas A, Wolchok JD, et al. Anti-programmed-death-receptor1 treatment with pembrolizumab in ipilimumab-refractory advanced melanoma: a randomised dose-comparison cohort of a phase 1 trial. Lancet 2014;384:1109-1117.

24. Topalian SL, Sznol M, McDermott DF, et al. Survival, durable tumor remission, and long-term safety in patients with advanced melanoma receiving nivolumab. J Clin Oncol 2014;32:1020-1030. 
25. Robert C, Schachter J, Long GV, et al. Pembrolizumab versus Ipilimumab in Advanced Melanoma. N Engl J Med 2015;372: 2521-2532.

26. Hamid O, Robert C, Daud A, et al. Safety and tumor responses with lambrolizumab (anti-PD-1) in melanoma. N Engl J Med 2013;369: 134-144.

27. Robert C, Long GV, Brady B, et al. Nivolumab in previously untreated melanoma without BRAF mutation. N Engl J Med 2015;372:320-330.

28. Larkin J, Chiarion-Sileni V, Gonzalez R, et al. Combined nivolumab and ipilimumab or monotherapy in untreated melanoma. N Engl J Med 2015;373:23-34

29. Brahmer JR, Tykodi SS, Chow LQ, et al. Safety and activity of anti-PD-L1 antibody in patients with advanced cancer. N Engl J Med 2012;366: 2455-2465.

30. Yang W, Chen PW, Li H, et al. PD-L1: PD-1 interaction contributes to the functional suppression of T-cell responses to human uveal melanoma cells in vitro. Invest Ophthalmol Vis Sci 2008;49:2518-2525.

31. Lee SJ, Lim HJ, Choi $\mathrm{YH}$, et al. The clinical significance of tumorinfiltrating lymphocytes and microscopic satellites in acral melanoma in a Korean population. Ann Dermatol 2013;25:61-66.

32. Song $H, W u Y$, Ren $G$, et al. Prognostic factors of oral mucosa melanoma: histopathological analysis in a retrospective cohort of 82 cases. Histopathology 2015;67:548-556.

33. Thierauf J, Veit JA, Affolter $A$, et al. Identification and clinical relevance of PD-L1 expression in primary mucosal malignant melanoma of the head and neck. Melanoma Res 2015;25:503-509.

34. Bonnelykke-Behrndtz ML, Steiniche T, Damsgaard TE, et al. MelanAnegative spindle-cell associated melanoma, a distinct inflammatory phenotype correlated with dense infiltration of CD163 macrophages and loss of E-cadherin. Melanoma Res 2015;25:113-118.

35. Kalluri R, Weinberg RA. The basics of epithelial-mesenchymal transition. J Clin Invest 2009;119:1420-1428.

36. Ock CY, Kim S, Keam B, et al. PD-L1 expression is associated with epithelial-mesenchymal transition in head and neck squamous cell carcinoma. Oncotarget 2016;7:15901-15914.

37. Kim S, Koh J, Kim MY, et al. PD-L1 expression is associated with epithelial-to-mesenchymal transition in adenocarcinoma of the lung. Hum Pathol 2016;58:7-14.

38. Rizvi NA, Hellmann MD, Snyder A, et al. Mutational landscape determines sensitivity to PD-1 blockade in non-small cell lung cancer. Science 2015;348:124-128.

39. Le DT, Uram JN, Wang $\mathrm{H}$, et al. PD-1 blockade in tumors with mismatch-repair deficiency. N Engl J Med 2015;372:2509-2520.

40. Shain $\mathrm{AH}$, Garrido $\mathrm{M}$, Botton $\mathrm{T}$, et al. Exome sequencing of desmoplastic melanoma identifies recurrent NFKBIE promoter mutations and diverse activating mutations in the MAPK pathway. Nat Genet 2015;47:1194-1199.
41. Hodis E, Watson IR, Kryukov GV, et al. A landscape of driver mutations in melanoma. Cell 2012;150:251-263.

42. Krauthammer $\mathrm{M}$, Kong $\mathrm{Y}, \mathrm{Ha} \mathrm{BH}$, et al. Exome sequencing identifies recurrent somatic RAC1 mutations in melanoma. Nat Genet 2012:44: 1006-1014.

43. Furney SJ, Turajlic S, Stamp G, et al. Genome sequencing of mucosal melanomas reveals that they are driven by distinct mechanisms from cutaneous melanoma. J Pathol 2013;230:261-269.

44. Furney SJ, Turajlic S, Stamp G, et al. The mutational burden of acral melanoma revealed by whole-genome sequencing and comparative analysis. Pigment Cell Melanoma Res 2014;27:835-838.

45. Van Raamsdonk CD, Griewank KG, Crosby MB, et al. Mutations in GNA11 in uveal melanoma. N Engl J Med 2010;363:2191-2199.

46. Davoli T, Uno H, Wooten EC, et al. Tumor aneuploidy correlates with markers of immune evasion and with reduced response to immunotherapy. Science 2017;355:6322.

47. Roh W, Chen PL, Reuben $\mathrm{A}$, et al. Integrated molecular analysis of tumor biopsies on sequential CTLA-4 and PD-1 blockade reveals markers of response and resistance. Sci Transl Med 2017:9:379.

48. Sautes-Fridman C, Lawand M, Giraldo NA, et al. Tertiary lymphoid structures in cancers: prognostic value, regulation, and manipulation for therapeutic intervention. Front Immunol 2016;7:407.

49. Eroglu Z, Kim DW, Johnson DB, et al. Response to anti-PD1/PDL1 therapy in patients with metastatic desmoplastic melanoma [ASCO abstract 9011]. J Clin Oncol 2015;33(15 Suppl):9011.

50. Niederkorn JY. Ocular immune privilege and ocular melanoma: parallel universes or immunological plagiarism? Front Immunol 2012;3: 148.

51. D'Angelo SP, Larkin J, Sosman JA, et al. Efficacy and safety of nivolumab alone or in combination with ipilimumab in patients with mucosal melanoma: a pooled analysis. J Clin Oncol 2017;35: 226-235.

52. Shoushtari AN, Munhoz RR, Kuk D, et al. The efficacy of anti-PD-1 agents in acral and mucosal melanoma. Cancer 2016;122:3354-3362.

53. Cho J, Ahn S, Yoo KH, et al. Treatment outcome of PD-1 immune checkpoint inhibitor in Asian metastatic melanoma patients: correlative analysis with PD-L1 immunohistochemistry. Invest New Drugs 2016;34:677-684

54. Herbst RS, Soria JC, Kowanetz M, et al. Predictive correlates of response to the anti-PD-L1 antibody MPDL3280A in cancer patients. Nature 2014;515:563-567.

55. Kottschade LA, Mcwilliams RR, Markovic SN, et al. The use of pembrolizumab for the treatment of metastatic uveal melanoma. Melanoma Res. 2016;26:300-303.

56. Algazi AP, Tsai KK, Shoushtari AN, et al. Clinical outcomes in metastatic uveal melanoma treated with PD-1 and PD-L1 antibodies. Cancer 2016;122:3344-3353. 